\title{
Damage detection based on mode shapes of a girder bridge constructed from responses of a moving vehicle under impact excitation
}

Zhongqiang Qi, Francis T. K. Au

Zhongqiang Qi, Francis T. K. Au, "Damage detection based on mode shapes of a girder bridge constructed from responses of a moving vehicle under impact excitation," Proc. SPIE 9805, Health Monitoring of Structural and Biological Systems 2016, 980507 (1 April 2016); doi: 10.1117/12.2218428

Event: SPIE Smart Structures and Materials + Nondestructive Evaluation and Health Monitoring, 2016, Las Vegas, Nevada, United States 


\title{
Damage detection based on mode shapes of a girder bridge constructed from responses of a moving vehicle under impact excitation
}

\author{
Zhongqiang QI*, Francis T. K. AU \\ Department of Civil Engineering, The University of Hong Kong, Pokfulam Road, Hong Kong, China
}

\begin{abstract}
The vibration mode shapes are often used to identify damage of bridges because the mode shapes are not only important modal properties but also sensitive to damage. However, the key issue is how to conveniently obtain the mode shapes of a bridge in service. Traditional methods invariably require installation of instruments on the bridge for collection of dynamic responses for constructing mode shapes, which are both costly and inconvenient. Therefore a method is developed to construct the mode shapes of simply supported bridges based on Hilbert Transform using only vehicle acceleration response for identification of the location of damage. Firstly, an algorithm is devised to construct the mode shapes by using the dynamic responses extracted from a moving vehicle under impact excitation. Then, based on these intermediate results, the coordinate modal assurance criterion in conjunction with suitable wavelets is used to identify the location of damage. Compared with the traditional methods, the proposed method uses only the information from the moving vehicle. Moreover, additional impact excitation on the vehicle helps to excite the bridge. This helps to improve the accuracy by overcoming the adverse effects of measurement noise and road surface roughness, which leads to high accuracy of damage detection. To verify the feasibility of the proposed method, some numerical studies have been carried out to investigate the effects of measurement noise, road surface roughness and multiple locations of damage on the accuracy of results.
\end{abstract}

Keywords: damage detection; impact excitation; measurement noise; mode shapes; moving vehicle; road surface roughness; vehicle-bridge interaction

\section{INTRODUCTION}

Damage detection of bridges has attracted much interest of researchers, especially by making use of mode shapes, which are not only important modal properties, but also provide a sensitive indicator for damage. Damage to a bridge leads to changes of mode shapes, which contain information of damage. Therefore, damage detection based on the mode shapes can identify not only the existence of damage, but also the location of damage. Zhang et al. ${ }^{1}$ presented a method to identify damage based on mode shape square extracted from a moving vehicle. Chen et al. ${ }^{2}$ proposed a method to detect damage based on optimized incomplete mode shape and frequency. Other researchers also used parameters related to mode shapes to identify damage, such as the slope ${ }^{3}$ and curvature ${ }^{4}$ of mode shape. However, the accuracy of these methods depends on the accuracy of mode shape obtained.

Traditional methods ${ }^{5}$ for constructing mode shapes often require a number of sensors to be installed at some important points of a bridge to pick up the time histories of dynamic responses. The selection of system identification or data processing techniques is of course crucial in the process. Moreover, to construct accurate mode shapes requires a large number of sensors, which will impose various limitations on practical application.

To overcome the excessive hardware requirements, Yang et al. ${ }^{6}$ presented an indirect method by using a test vehicle to move on a simply supported bridge. Then Hilbert transform was applied to the measured vehicle acceleration response to extract the mode shapes associated with various bridge frequencies. After examining the effects of several factors on the accuracy of results, including the vehicle speed, random traffic and road surface roughness, the road surface roughness was found to affect the accuracy seriously. Some other researchers ${ }^{7,8}$ also proposed damage detection methods similar to this indirect method.

Zhang et al. ${ }^{9}$ attempted to obtain the deflection shape curvature of beam and plate like structures from the dynamic response of a passing vehicle excited by a tapping force comprising a series of sinusoidal exciting forces. They used the

Health Monitoring of Structural and Biological Systems 2016, edited by Tribikram Kundu,

Proc. of SPIE Vol. 9805, 980507 · @ 2016 SPIE · CCC code: 0277-786X/16/\$18 · doi: 10.1117/12.2218428 
response of test vehicle to construct the approximate modal shape squares of the structure. Based on that, damage detection could be carried out.

The present study is to identify the location of damage based on the mode shapes of a simply supported bridge constructed by using the dynamic response extracting from a moving vehicle under impact excitation. To reduce the adverse effects of road surface roughness and measurement noise, an impact excitation is applied on the vehicle to help excite the bridge. By a comprehensive numerical study, the feasibility of the present method is evaluated taking into account the measurement noise, road surface roughness and multiple locations of damage.

\section{METHODOLOGY}

\subsection{Construction of mode shapes of a simply supported bridge}

The simply supported bridge being studied is modelled as an Euler-Bernoulli beam and the vehicle is modelled as a sprung mass as shown in Figure 1. The stiffness of spring between bridge and vehicle body is $k_{v}$, while the mass of vehicle is $m_{v}$.

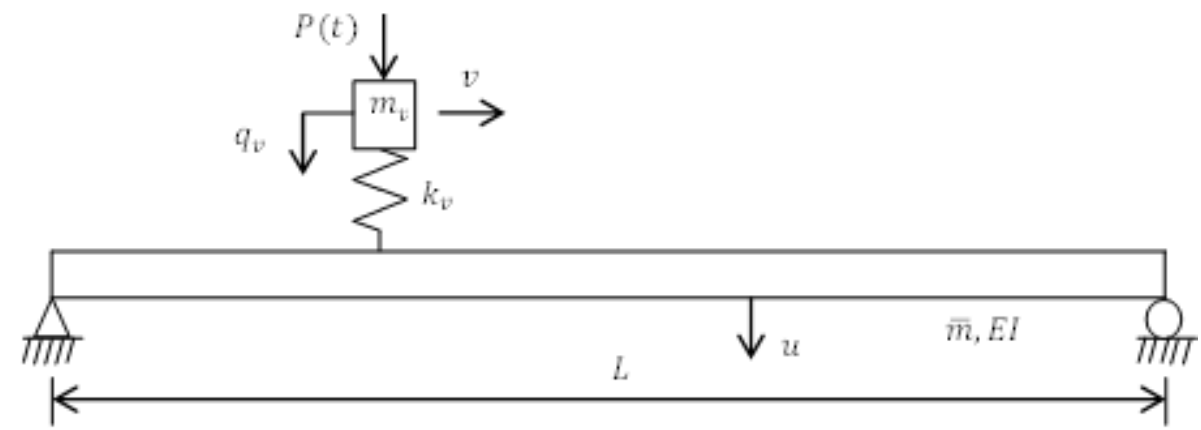

Figure 1. Vehicle bridge interaction system

Neglecting the damping of the bridge and vehicle, the equation of motion for the bridge and vehicle can be written respectively as:

$$
\begin{gathered}
\bar{m} \ddot{u}(x, t)+E I u^{\prime \prime \prime \prime}(x, t)=f_{c}(t) \delta(x-v t) \\
m_{v} \ddot{q}_{v}+k_{v}\left(q_{v}-\left.u\right|_{x=v t}\right)=P(t) \\
f_{c}(t)=-m_{v} g+k_{v}\left(q_{v}-\left.u\right|_{x=v t}\right)
\end{gathered}
$$

where $\bar{m}$ and $E I$ are the mass per unit length and the flexural rigidity of the bridge respectively; $u(x, t)$ and $q_{v}$ represent the vertical displacements of the bridge and vehicle respectively; the dot and prime denote differentiation with respect to time $t$ and coordinate $x$ respectively; $P(t)$ is the external force applied on the vehicle; $\delta$ is the delta function; and $f_{c}(t)$ is the contact force between the spring and bridge surface.

The displacement response of the bridge of span $L$ can be expressed in terms of the mode shapes $\varphi_{n}(x)$ and modal coordinates $y_{b, n}(t)$ by modal superposition method as

$$
u(x, t)=\sum_{n} \varphi_{n}(x) * y_{b, n}(t)
$$

Eq. (1) and Eq. (2) can be solved by using time-stepping method with a time interval of $\Delta t=t_{i+1}-t_{i}$ for $i=1$ to $N$. After getting the solution to Eq. (1), the bridge response is substituted into Eq. (2), giving the acceleration response of vehicle at time $t_{i+1}$ as:

$$
\begin{gathered}
\ddot{q}_{v}\left(t_{i+1}\right)=A 1(i) * \cos \left(\omega_{v} \Delta t\right)+B 1(i) * \sin \left(\omega_{v} \Delta t\right)+\frac{P(t)}{m_{v} \omega_{v}^{2}} \\
+\sum_{n} C 1(n, i) * \varphi_{n}\left(v t_{i}\right) \cos \left[\omega_{b, n} \Delta t\right]+C 2(n, i) * \varphi_{n}\left(v t_{i}\right) \sin \left[\omega_{b, n} \Delta t\right]
\end{gathered}
$$




$$
+\ddot{G}\left[\varphi_{n}\left(v t_{i+1}\right), \omega_{b, n}\right]
$$

where $G$ is a function depending on the bridge response and mode shape but independent of the bridge frequencies; the first group of coefficients are

$$
\begin{gathered}
C 1(n, i)=-\omega_{b, n}{ }^{2} * A(n, i) * \frac{\omega_{v}^{2}}{\omega_{v}^{2}-\omega_{b, n}{ }^{2}} \\
C 2(n, i)=-\omega_{b, n}{ }^{2} * B(n, i) * \frac{\omega_{v}^{2}}{\omega_{v}^{2}-\omega_{b, n}{ }^{2}} \\
A 1(i)=\left\{q_{v}\left(t_{i}\right)-\sum_{n} C 1(n, i) / \omega_{v}^{2}+G\left[\varphi_{n}\left(v t_{i}\right), \omega_{b, n}\right]-\frac{P\left(t_{i}\right)}{m_{v} * \omega_{v}^{2}}\right\} \\
B 11(i)=\left\{\dot{q}_{v}\left(t_{i}\right)-\sum_{n} C 2(n, i) / \omega_{v}+\dot{G}\left[\varphi_{n}\left(v t_{i}\right), \omega_{b, n}\right]\right\} / \omega_{v}
\end{gathered}
$$

the second group of coefficients are

$$
\begin{gathered}
C(n, i)=-\frac{2\left(m_{v} g+m_{v} \ddot{q}_{v}\left(t_{i}\right)+P\left(t_{i}\right)\right)}{\bar{m} T\left(\omega_{b, n}^{2}-\beta_{n}^{2} v^{2}\right)} \\
A(n, i)=y_{b, n}\left(t_{i}\right)-C(n, i) * F\left[\varphi_{n}\left(v t_{i}\right), \omega_{b, n}\right] \\
B(n, i)=\frac{\left(y_{b, n}\left(t_{i}\right)-v \beta_{n} C(n, i) * \dot{F}\left[\varphi_{n}\left(v t_{i}\right), \omega_{b, n}\right]\right)}{\omega_{b, n}}
\end{gathered}
$$

$v$ is the speed of vehicle; $T=\int_{0}^{L} \varphi_{n}(x) * \varphi_{n}{ }^{T}(x) d x$; the $n$th natural frequency of the bridge is $\omega_{b, n}=\beta_{n}^{2} \sqrt{\frac{E I}{\bar{m}}}$; the frequency of the vehicle is $\omega_{v}=\sqrt{\frac{k_{v}}{m_{v}}} ; \beta_{n}$ is a parameter associated with the $n^{\text {th }}$ mode shapes; and $F\left[\varphi_{n}\left(v t_{i+1}\right), \omega_{b, n}\right]$ is a function of the mode shapes based on the right side of Eq. (1).

From Eq. (5), the vehicle acceleration response at time $t_{i+1}$ is related to that at time $t_{i}$. Therefore, if the initial conditions, e.g. $u\left(x, t_{0}\right)=0$ and $\ddot{q}_{v}\left(t_{0}\right)$, are known, the vehicle acceleration response can be calculated by using time stepping method.

A suitable filter can be applied to the vehicle acceleration response to extract the component response $Q(n, i)$ associated with each bridge frequency

$$
Q(n, i)=C 1(n, i) * \varphi_{n}\left(v t_{i}\right) \cos \left[\omega_{b, n} \Delta t\right]+C 2(n, i) * \varphi_{n}\left(v t_{i}\right) \sin \left[\omega_{b, n} \Delta t\right]
$$

Using Hilbert Transform ${ }^{10}$, its transform is obtained as

$$
\hat{Q}(n, i)=C 1(n, i) * \varphi_{n}\left(v t_{i}\right) \sin \left[\omega_{b, n} \Delta t\right]-C 2(n, i) * \varphi_{n}\left(v t_{i}\right) \cos \left[\omega_{b, n} \Delta t\right]
$$

The instantaneous amplitude of the transform pair of $Q$ can be obtained as

$$
T(i)=\sqrt{Q(n, i)^{2}+\hat{Q}(n, i)^{2}}=\sqrt{2\left(C 1^{2}(n, i)+C 2^{2}(n, i)\right) \varphi_{n}^{2}\left(v t_{i}\right)}
$$

Replacing $v t_{i}$ with $x_{t_{i}}$ and re-arranging, one has

$$
\left|\varphi_{n}\left(x_{t_{i}}\right)\right|=\frac{T(i)}{\sqrt{2\left(C 1^{2}(n, i)+C 2^{2}(n, i)\right.}}
$$

The above equation shows that the $n^{\text {th }}$ mode shape is related to coefficients $C 1$ and $C 2$ that can be obtained from the vehicle acceleration response. Hence, the mode shape can be identified according to Eq. (11). 


\subsection{Damage detection based on mode shape}

Based on the mode shapes obtained from Section 2.1, two methods are adopted here to identify the location of damage.

Method 1: Wavelet Transform

Let $W f(s, a)$ be the continuous wavelet transform of a function $f(u)$ for scale parameter $s$ and translation parameter $a$.

The location of a wavelet transform modulus maximum can be determined as $\left(s_{0}, a_{0}\right)$ by having $\frac{\partial W f\left(s_{0}, a_{0}\right)}{\partial a}=0$. Damage can lead to stiffness reduction of a bridge and hence mode shape mutation with the mutation points appearing as singular points. According to the wavelet transform modulus maxima method, the wavelet transform coefficients display peak values at the singular points, which are regarded as potential damage locations.

Method 2: Co-Ordinate Modal Assurance Criterion (COMAC)

Another damage indicator to identify the location of damage is $C O M A C,{ }^{11}$ which can be defined as

$$
\operatorname{COMAC}(i)=\frac{\left[\sum_{j=1}^{N}\left|\varphi_{i, j}^{T} \varphi_{i, j}^{P}\right|\right]^{2}}{\sum_{j}^{N}\left(\varphi_{i, j}^{T}\right)^{2} \sum_{j}^{N}\left(\varphi_{i, j}^{P}\right)^{2}}
$$

where $\varphi_{i, j}^{T}$ and $\varphi_{i, j}^{P}$ are the $j^{\text {th }}$ mode shape amplitudes of the intact and damaged bridge at point $i$. For perfect mode shapes of a bridge without damage, $\operatorname{COMAC}(i)=1$.

\subsection{Procedure of identifying location of damage}

The following steps show the procedure of damage detection from the vehicle acceleration response.

(a) To obtain the acceleration response of a moving vehicle when it passes over the bridge

For numerical simulation, the response is calculated by finite element method or similar. For experimental studies, the response is measured from the sensors on the vehicle.

(b) To identify the natural frequencies of the bridge

The vehicle acceleration response will be processed by a suitable identification method such as Fast Fourier Transform (FFT) to obtain the natural frequencies of the bridge.

(c) To extract the component response associated with a bridge frequency from the vehicle acceleration

A suitable filter will be selected to extract the history of component response associated with each bridge natural frequency. In this study, the band-pass filter is selected.

(d) To construct the mode shapes associated with the selected frequencies

(e) To identify the location of damage by using wavelet transform and COMAC.

\section{CASE STUDY}

The proposed method is verified with a simply-supported bridge with a span of $L=30 \mathrm{~m}$, mass per unit length of $\bar{m}=$ $1000 \mathrm{~kg} / \mathrm{m}$ and flexural rigidity of $E I=2.2 \times 10^{10} \mathrm{Nm}^{2}$. The moving vehicle has a mass of $m_{v}=1000 \mathrm{~kg}$, and suspension stiffness of $k_{v}=170000 \mathrm{kN} / \mathrm{m}$. The bridge is divided into 30 identical elements for analysis of vehicle-bridge interaction with time steps of $0.001 \mathrm{~s}$. The impact excitation is simulated as an impulse resulting from a drop mass. The damage is simulated as reduction of element stiffness.

\subsection{Effect of multiple locations of damage}

Both the cases of single and multiple locations of damage are considered as shown in Table 1. The impact excitation is taken as a rectangular impulse associated with a mass of $m_{d}=200 \mathrm{~kg}$ dropping through a height of $h=0.3 \mathrm{~m}$. The drop mass is released when the vehicle travelling right from the left end at a speed $v=2 \mathrm{~m} / \mathrm{s}$ reaches a position $6 \mathrm{~m}$ from the left end of bridge. The simulated vehicle acceleration response is calculated from finite element method by neglecting measurement noise and road surface roughness in this section. Then the bridge frequencies are identified by using FFT. A 
suitable band-pass filter is selected to extract the component responses associated with the first three frequencies. Because of the absence of strong signals prior to impact excitation, the moving vehicle has to run over the bridge twice in two different directions so that complete mode shapes can be identified by combination. After normalization and calculating the related parameters, the whole mode shapes can be constructed as shown in Figure. 2.

Table 1. Cases of damage

\begin{tabular}{|c|c|c|}
\hline Cases & Element number(s) of damage & Stiffness Reduction (\%) \\
\hline 1 & 6 & 20 \\
\hline 2 & 6 and 12 & 20 and 30 \\
\hline
\end{tabular}
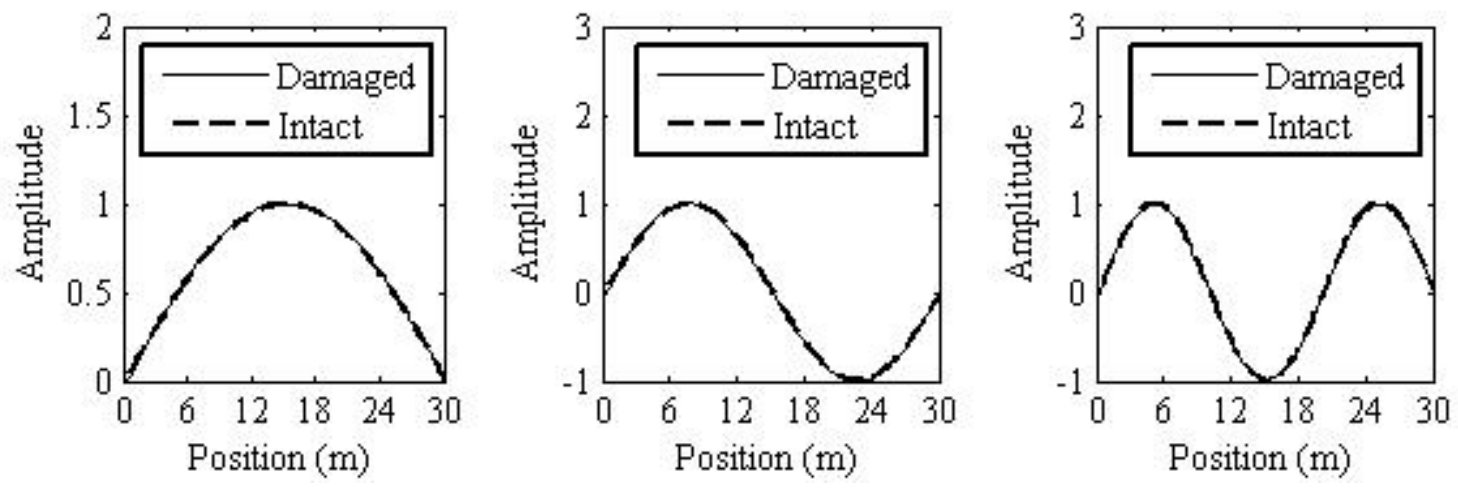

(a) Case 1 of damage
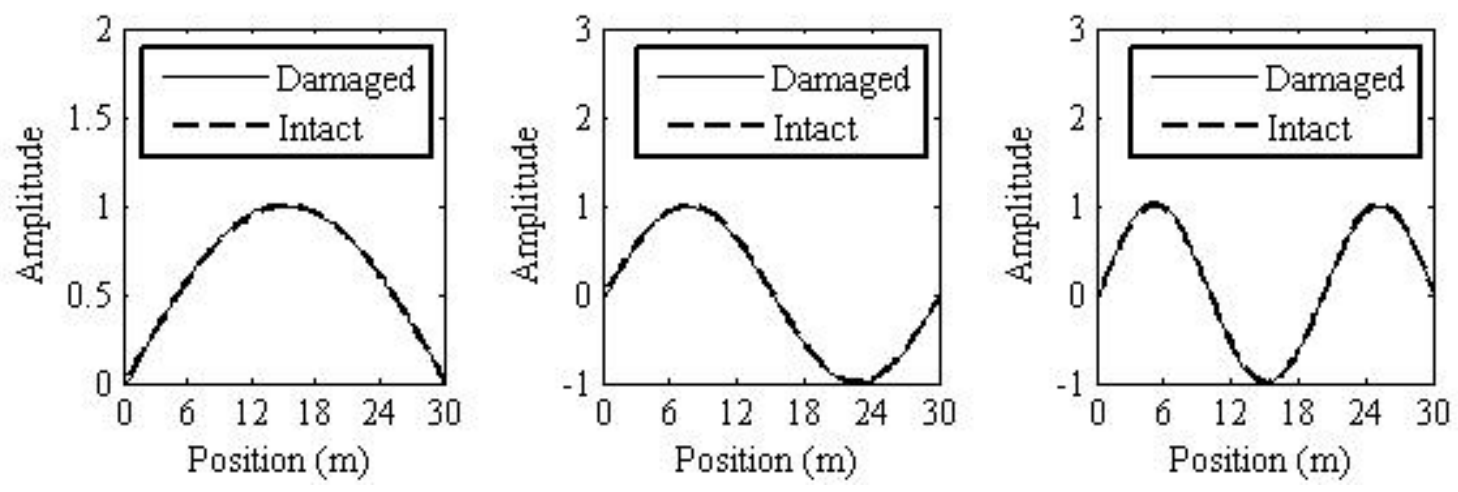

(b) Case 2 of damage

Figure 2. First three mode shapes constructed for intact and damaged scenarios of bridge without road surface roughness and measurement noise

Figure 3 shows the wavelet coefficients calculated from the first three mode shapes for the two cases by using Mexican wavelet. In the charts, the true damage location is indicated by two vertical lines. Clearly, one location or multiple of damage can be identified from the wavelet coefficients of the constructed mode shapes, although the clarity varies case to case. However the local peaks adjacent to supports have to be interpreted with care, because of their proximity to hinge supports. Table 2 shows the COMAC values calculated from the constructed mode shapes of the intact and scenarios, where the nodes are numbered sequentially from the left end of bridge. Although COMAC can also identify location of damage, it may not be as obvious. 

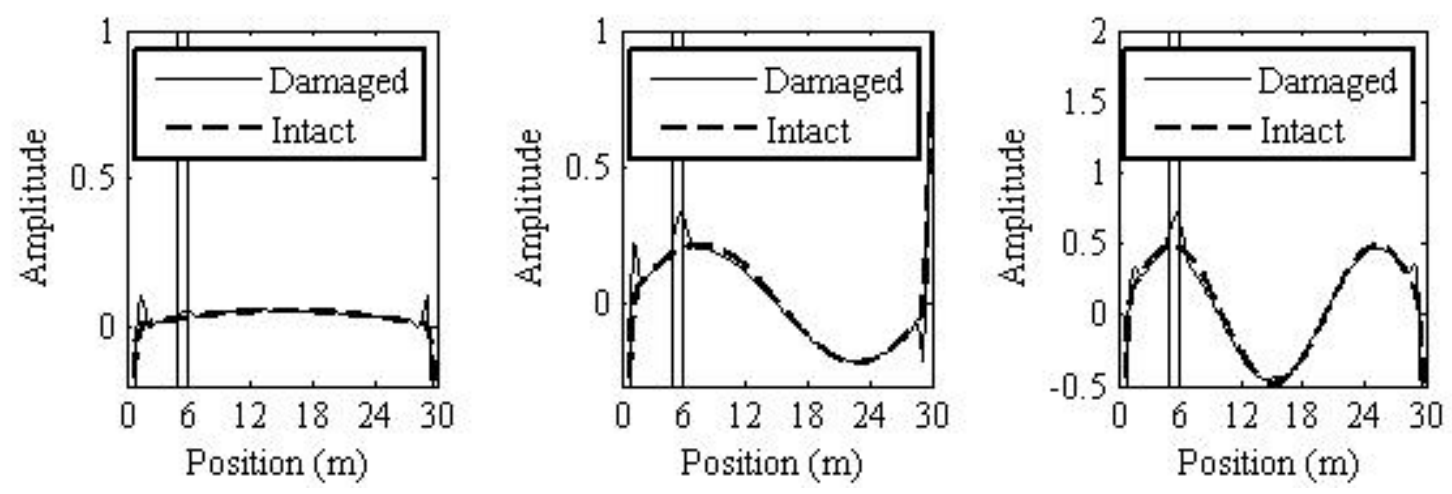

(a) Case 1 of damage
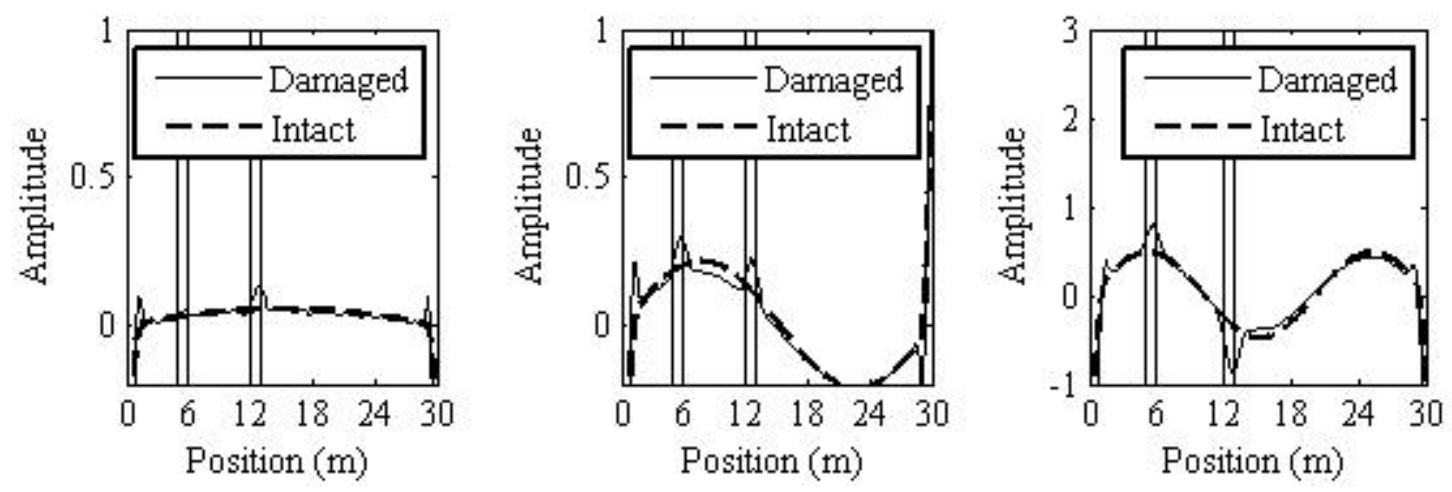

(b) Case 2 of damage

Figure 3. Wavelet coefficients obtained from the first three mode shapes constructed for intact and damaged scenarios of bridge without road surface roughness and measurement noise

Table 2. Values of COMAC for the two damage cases of bridge without road surface roughness and measurement noise

\begin{tabular}{|c|c|c|c|c|c|c|c|c|c|c|c|}
\hline Node & $\mathbf{1}$ & $\mathbf{2}$ & $\mathbf{3}$ & $\mathbf{4}$ & $\mathbf{5}$ & $\mathbf{6}$ & $\mathbf{7}$ & $\mathbf{8}$ & $\mathbf{9}$ & $\mathbf{1 0}$ & $\mathbf{1 1}$ \\
\hline Case 1 & -- & 0.993 & 1.004 & 0.991 & 0.996 & 0.950 & 0.947 & 1.006 & 1.004 & 0.996 & 1.009 \\
\hline Case 2 & -- & 1.006 & 1.008 & 0.993 & 1.008 & 0.951 & 0.953 & 0.996 & 1.001 & 1.009 & 1.009 \\
\hline Node & $\mathbf{1 2}$ & $\mathbf{1 3}$ & $\mathbf{1 4}$ & $\mathbf{1 5}$ & $\mathbf{1 6}$ & $\mathbf{1 7}$ & $\mathbf{1 8}$ & $\mathbf{1 9}$ & $\mathbf{2 0}$ & $\mathbf{2 1}$ & $\mathbf{2 2}$ \\
\hline Case 1 & 0.991 & 0.999 & 0.998 & 1.005 & 1.006 & 0.994 & 1.000 & 0.999 & 1.003 & 1.004 & 1.005 \\
\hline Case 2 & 0.993 & 0.944 & 0.945 & 1.000 & 1.006 & 0.993 & 0.998 & 1.008 & 1.006 & 1.009 & 1.003 \\
\hline Node & $\mathbf{2 3}$ & $\mathbf{2 4}$ & $\mathbf{2 5}$ & $\mathbf{2 6}$ & $\mathbf{2 7}$ & $\mathbf{2 8}$ & $\mathbf{2 9}$ & $\mathbf{3 0}$ & $\mathbf{3 1}$ & & \\
\hline Case 1 & 0.996 & 1.004 & 1.003 & 0.993 & 0.992 & 1.000 & 1.009 & 0.997 & -- & & \\
\hline Case 2 & 0.991 & 1.007 & 1.009 & 1.004 & 1.005 & 1.005 & 0.998 & 1.003 & -- & & \\
\hline
\end{tabular}

\subsection{Effect of road surface roughness}

Different degrees of road surface roughness are considered when constructing the mode shapes of a bridge carrying a vehicle travelling at a speed $v=2 \mathrm{~m} / \mathrm{s}$. The road surface roughness is classified as one of the five degrees according to 
IOS8608. Damage Case 2 in Table 1 is used in this section. The identified mode shapes are broadly similar to those in Section 3.1.

Figure 4 shows the wavelet coefficients calculated from the constructed mode shapes for different degrees of road surface roughness by using Mexican Hat wavelet. Clearly, the impact excitation helps to excite the bridge and improve the accuracy of mode shapes. However, when the road surface roughness reaches the bad level, the location of damage can hardly be identified through the second and third mode shapes. In other words, the higher order mode shapes are more affected by poor road surface conditions. Table 3 shows the COMAC values calculated from the constructed mode shapes of the intact and damaged scenarios. When the road surface roughness is worse than the average level, COMAC cannot identify the location of damage well. Higher impact excitation should be applied on the vehicle to overcome this problem.
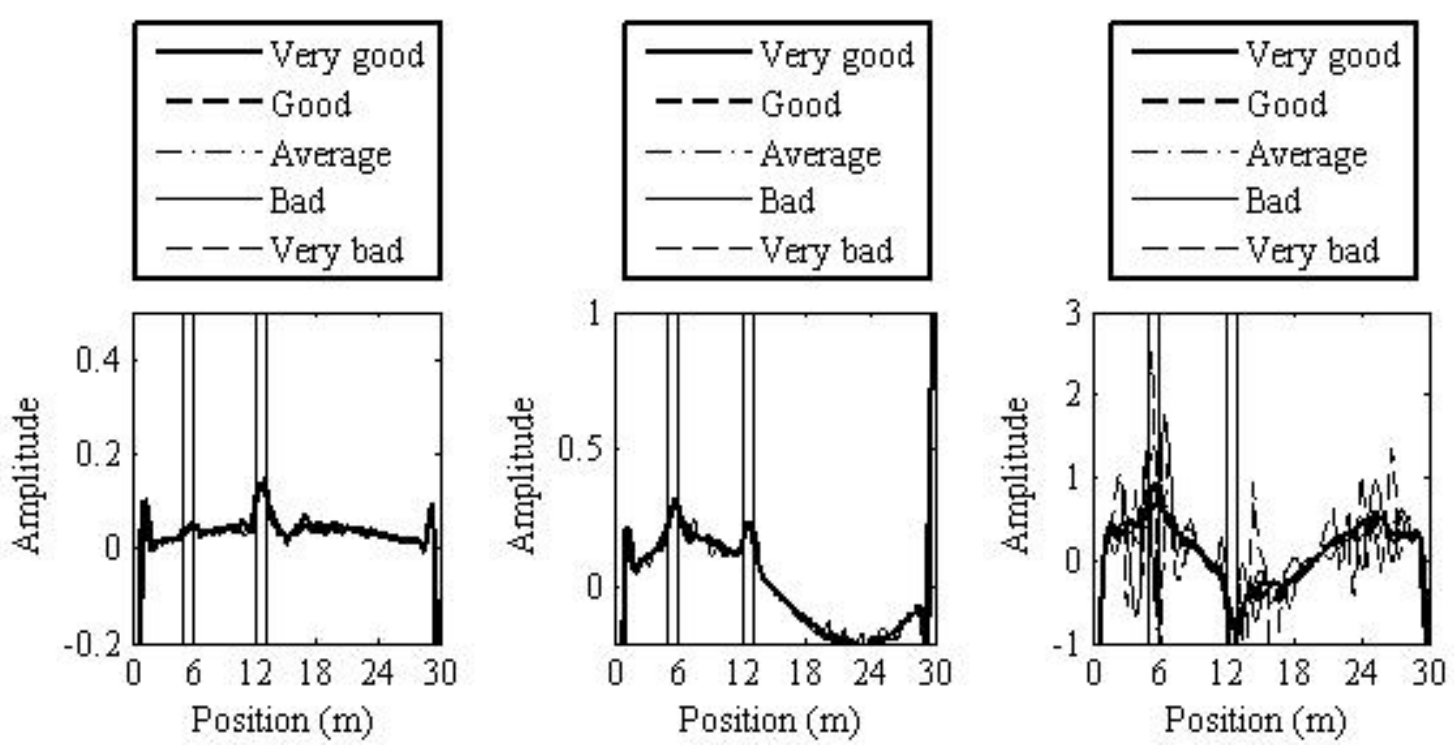

Figure 4. Wavelet coefficients obtained from the first three mode shapes constructed for damaged bridge with road surface roughness but without measurement noise 
Table 3.Values of COMAC for damage Case 2 of bridge with road surface roughness but without measurement noise

\begin{tabular}{|c|c|c|c|c|c|c|c|c|c|c|c|}
\hline Node & $\mathbf{1}$ & $\mathbf{2}$ & $\mathbf{3}$ & $\mathbf{4}$ & $\mathbf{5}$ & $\mathbf{6}$ & $\mathbf{7}$ & $\mathbf{8}$ & $\mathbf{9}$ & $\mathbf{1 0}$ & $\mathbf{1 1}$ \\
\hline Very good & -- & 1.006 & 1.009 & 0.991 & 1.007 & 0.950 & 0.954 & 0.997 & 1.000 & 1.009 & 1.007 \\
\hline Good & -- & 1.011 & 1.013 & 0.989 & 1.006 & 0.952 & 0.950 & 0.986 & 1.005 & 1.008 & 1.012 \\
\hline Average & -- & 0.982 & 0.961 & 1.031 & 1.033 & 0.950 & 0.943 & 0.972 & 1.033 & 1.021 & 0.969 \\
\hline Bad & -- & 0.973 & 0.969 & 1.028 & 1.031 & 0.949 & 0.946 & 0.977 & 0.967 & 0.971 & 1.029 \\
\hline Very bad & -- & 0.952 & 0.978 & 1.039 & 1.069 & 0.948 & 0.941 & 0.984 & 0.956 & 0.967 & 1.042 \\
\hline Node & $\mathbf{1 2}$ & $\mathbf{1 3}$ & $\mathbf{1 4}$ & $\mathbf{1 5}$ & $\mathbf{1 6}$ & $\mathbf{1 7}$ & $\mathbf{1 8}$ & $\mathbf{1 9}$ & $\mathbf{2 0}$ & $\mathbf{2 1}$ & $\mathbf{2 2}$ \\
\hline Very good & 0.992 & 0.945 & 0.941 & 1.001 & 1.003 & 0.994 & 0.997 & 1.003 & 1.006 & 1.006 & 1.002 \\
\hline Good & 0.985 & 0.942 & 0.943 & 1.011 & 1.004 & 0.990 & 0.984 & 1.016 & 0.989 & 0.985 & 1.009 \\
\hline Average & 1.031 & 0.946 & 0.947 & 1.027 & 0.988 & 1.013 & 1.019 & 1.018 & 1.026 & 1.007 & 0.981 \\
\hline Bad & 1.039 & 0.944 & 0.949 & 1.037 & 0.983 & 0.974 & 0.981 & 1.021 & 1.019 & 1.030 & 1.018 \\
\hline Very bad & 1.021 & 0.941 & 0.951 & 1.028 & 0.963 & 0.969 & 0.989 & 1.029 & 1.038 & 1.074 & 0.959 \\
\hline Node & $\mathbf{2 3}$ & $\mathbf{2 4}$ & $\mathbf{2 5}$ & $\mathbf{2 6}$ & $\mathbf{2 7}$ & $\mathbf{2 8}$ & $\mathbf{2 9}$ & $\mathbf{3 0}$ & $\mathbf{3 1}$ & & \\
\hline Very good & 0.993 & 1.009 & 1.007 & 1.002 & 1.005 & 1.003 & 0.999 & 1.004 & -- & & \\
\hline Good & 1.013 & 1.006 & 1.010 & 1.003 & 1.009 & 1.010 & 0.990 & 1.012 & -- & & \\
\hline Average & 0.977 & 1.021 & 1.013 & 0.985 & 0.981 & 0.979 & 1.028 & 1.017 & -- & & \\
\hline Bad & 0.982 & 1.019 & 1.028 & 1.017 & 1.007 & 1.019 & 0.983 & 0.976 & -- & & \\
\hline Very bad & 0.976 & 1.051 & 1.084 & 1.031 & 0.991 & 1.021 & 1.014 & 1.034 & -- & & \\
\hline
\end{tabular}

\subsection{Effect of measurement noise}

Different degrees of signal-noise ratio (SNR) are then considered when constructing the mode shapes of a bridge carrying a vehicle travelling at a speed $v=2 \mathrm{~m} / \mathrm{s}$. Damage Case 2 is considered in this Section. The identified mode shapes are broadly similar to those in Section 3.1.

Figure 5 shows the wavelet coefficients calculated from the constructed mode shapes for different SNRs. Clearly, the impact excitation helps to excite the bridge and improve the accuracy of mode shapes. Table 4 shows the COMAC values calculated from the constructed mode shapes of the intact and damaged scenarios. However, when SNR is above $10 \%$, the location of damage can hardly be identified through the mode shapes. Higher impact excitation should be applied on the vehicle to overcome this problem. 

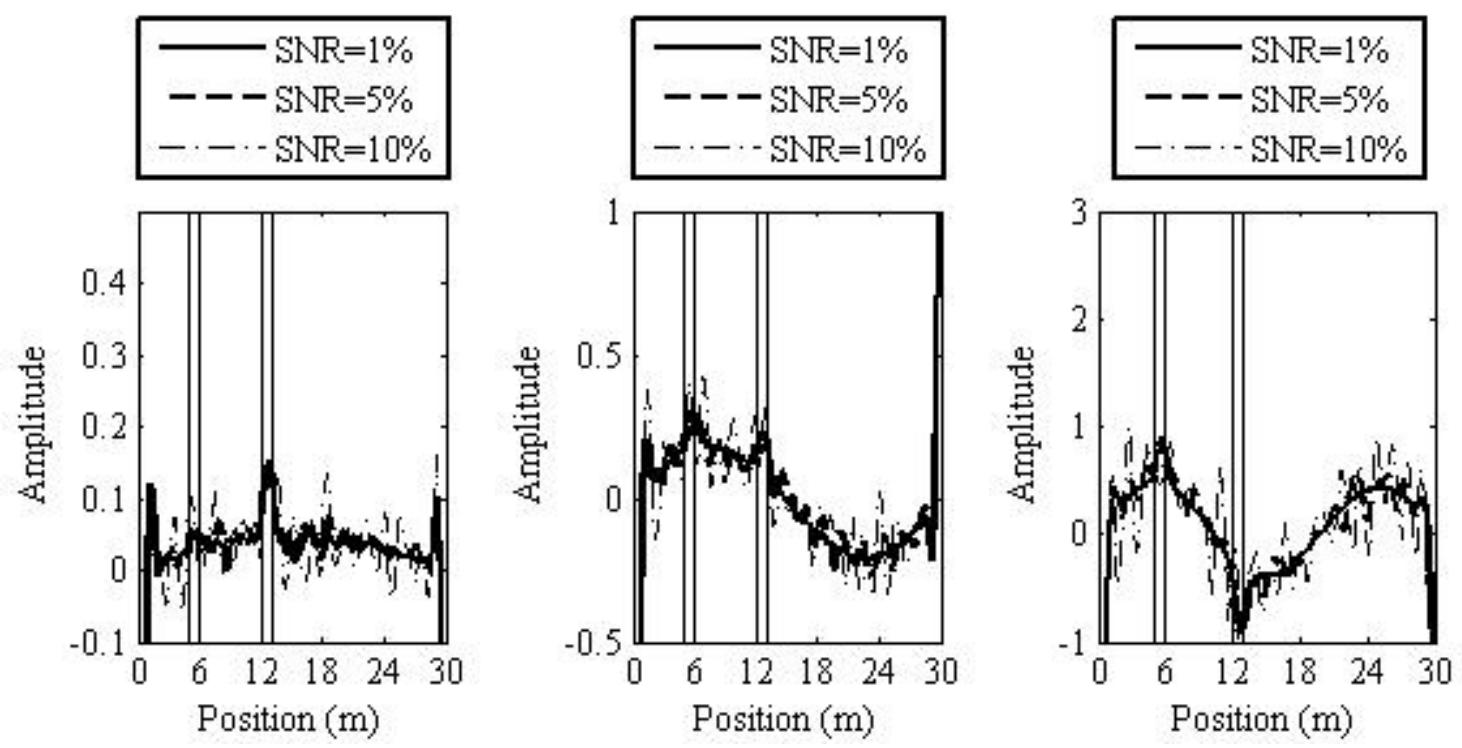

Figure 5. Wavelet coefficients obtained from the first three mode shapes constructed for damaged bridge without road surface roughness but with measurement noise

Table 4.Value of COMAC for damage Case 2 of bridge without road surface roughness but with measurement noise

\begin{tabular}{|c|c|c|c|c|c|c|c|c|c|c|c|}
\hline Node & $\mathbf{1}$ & $\mathbf{2}$ & $\mathbf{3}$ & $\mathbf{4}$ & $\mathbf{5}$ & $\mathbf{6}$ & $\mathbf{7}$ & $\mathbf{8}$ & $\mathbf{9}$ & $\mathbf{1 0}$ & $\mathbf{1 1}$ \\
\hline $\mathrm{SNR}=1 \%$ & -- & 1.002 & 1.005 & 0.996 & 0.999 & 0.951 & 0.955 & 0.998 & 1.003 & 1.006 & 1.004 \\
\hline $\mathrm{SNR}=5 \%$ & -- & 0.990 & 0.987 & 1.011 & 0.987 & 0.948 & 0.952 & 1.006 & 0.998 & 0.986 & 0.986 \\
\hline $\mathrm{SNR}=10 \%$ & -- & 1.032 & 0.979 & 1.046 & 1.022 & 0.955 & 0.959 & 0.967 & 0.980 & 1.018 & 0.954 \\
\hline Node & $\mathbf{1 2}$ & $\mathbf{1 3}$ & $\mathbf{1 4}$ & $\mathbf{1 5}$ & $\mathbf{1 6}$ & $\mathbf{1 7}$ & $\mathbf{1 8}$ & $\mathbf{1 9}$ & $\mathbf{2 0}$ & $\mathbf{2 1}$ & $\mathbf{2 2}$ \\
\hline $\mathrm{SNR}=1 \%$ & 0.997 & 0.941 & 0.948 & 1.002 & 1.005 & 0.996 & 0.998 & 1.004 & 1.001 & 1.003 & 1.005 \\
\hline $\mathrm{SNR}=5 \%$ & 1.010 & 0.945 & 0.950 & 0.985 & 0.990 & 1.010 & 1.002 & 0.987 & 0.991 & 0.986 & 0.995 \\
\hline $\mathrm{SNR}=10 \%$ & 1.046 & 0.951 & 0.948 & 0.973 & 0.970 & 1.031 & 1.001 & 1.005 & 0.985 & 0.979 & 0.974 \\
\hline $\mathrm{Node}$ & $\mathbf{2 3}$ & $\mathbf{2 4}$ & $\mathbf{2 5}$ & $\mathbf{2 6}$ & $\mathbf{2 7}$ & $\mathbf{2 8}$ & $\mathbf{2 9}$ & $\mathbf{3 0}$ & $\mathbf{3 1}$ & & \\
\hline $\mathrm{SNR}=1 \%$ & 0.997 & 0.099 & 1.006 & 1.001 & 1.003 & 1.004 & 0.998 & 1.002 & -- & & \\
\hline $\mathrm{SNR}=5 \%$ & 1.013 & 0.989 & 0.986 & 0.994 & 0.992 & 0.992 & 1.003 & 0.995 & -- & & \\
\hline $\mathrm{S} N \mathrm{~N}=10 \%$ & 1.022 & 0.082 & 0.984 & 1.033 & 1.038 & 1.016 & 0.954 & 1.015 & -- & & \\
\hline
\end{tabular}

\section{CONCLUSIONS}

An indirect method is developed to identify the location of damage by using mode shapes of a simply supported bridge extracted from the acceleration response of a vehicle moving on the bridge. An additional impact excitation is applied on the vehicle to help excite the bridge. The numerical study shows that the impact excitation helps to overcome the adverse effect of road surface roughness and measurement noise for construction of accurate mode shapes from the vehicle response and improve the accuracy of damage detection. 


\section{REFERENCES}

[1] Zhang, Y., Wang, L. and Xiang, Z., "Damage detection by mode shape squares extracted from a passing vehicle," J. Sound Vib. Tech. Papers 331(2), 291-307 (2012).

[2] Chen, W., Zhao, W. G., Yang., H. Z. and Chen, X. Q., "Damage detection based on optimized incomplete mode shape and frequency," Acta Mechanica Solida Sinica. Tech. Papers 28(1), 74-82 (2015).

[3] Zhu, H. P., Lin, L. and He, X. Q., "Damage detection method for shear buildings using the changes in the first mode shape slopes," Computers and Structures. Tech. Papers 89(9), 733-743 (2011).

[4] Dessi, D. and Camerlengo, G., "Damage identification techniques via modal curvature analysis: Overview and comparison," Mechanical Systems and Signal Processing. Tech. Papers 52, 181-205 (2015).

[5] Farrar, C. R. and James III, G. H., "System identification from ambient vibration measurements on a bridge," J. Sound Vib. Tech. Papers 205(1), 1-18 (1997).

[6] Yang, Y. B., Li, Y. C. and Chang, K. C., "Constructing the mode shapes of a bridge from a passing vehicle: a theoretical study," Smart Structures and Systems. Tech. Papers 13(5), 797-819 (2014).

[7] Bu, J. Q., Law, S. S. and Zhu, X. Q., "Innovative bridge condition assessment from dynamic response of a passing vehicle," J. Eng. Mech. - ASCE. Tech. Papers 132(12), 1372-1379 (2006).

[8] McGetrick, P. J., Gonzalez, A. and Obrien, E. J., "Theoretical investigation of the use of a moving vehicle to identify bridge dynamic parameters," Insight. Tech. Papers 51(8), 433-438 (2009).

[9] Zhang, Y. Lie, T. and Xiang, Z., "Damage detection method based on operating deflection shape curvature extracted from dynamic response of a passing vehicle," J. Mechanical Systems and Signal Processing. Tech. Papers 35, 238-254 (2013).

[10] Huang, N. E., Shen, Z., Long, S. R., Wu, M. C., Shih, H. H., Zheng, Q., Yeh, N. C., Tung, C. C. and Liu, H. H., "The empirical mode decomposition and the Hilbert spectrum for nonlinear and non-stationary time series analysis," Mathematical. Physical and Engineering Sciences. Tech. Papers 454, 903-995 (1998).

[11] West, W. M., "Illustration of the use of modal assurance criterion to detect structural changes in an orbit test specimen,” Proc. International Modal Analysis Conference 1, 1-6 (1986). 